\title{
Linx
}

Revue des linguistes de l'université Paris X Nanterre

De la « Déclaration des droits de l'homme et du citoyen » (1789) à la « Déclaration universelle des droits de l'homme » (1948) constantes et changements

\section{Geneviève Petiot}

\section{OpenEdition}

\section{Journals}

Édition électronique

URL : http://journals.openedition.org/linx/225

DOI : $10.4000 /$ linx.225

ISSN : 2118-9692

Éditeur

Presses universitaires de Paris Nanterre

Édition imprimée

Date de publication : 1 juin 2005

Pagination : 139-153

ISSN : 0246-8743

Référence électronique

Geneviève Petiot, « De la « Déclaration des droits de l'homme et du citoyen » (1789) à la « Déclaration universelle des droits de l'homme » (1948) constantes et changements », Linx [En ligne], 52 | 2005, mis en ligne le 31 janvier 2011, consulté le 10 décembre 2020. URL : http://journals.openedition.org/linx/ 225 ; DOI : https://doi.org/10.4000/linx.225 


\title{
De la « Déclaration des droits de l'homme et du citoyen » (1789) à la « Déclaration universelle des droits de l'homme " (1948) constantes et changements
}

\author{
Geneviève Petiot
}

SYLED - CEDISCOR - Université Paris III - Sorbonne Nouvelle

L'étude proposée ici de ces deux discours politiques se limite à la confrontation des articles des deux Déclarations traitant des «droits de l'homme (et du citoyen)», à partir de quelques vocables, ceux qui organisent les intitulés de ces deux Déclarations; elle devrait permettre de les situer l'un par rapport à l'autre, mais aussi par rapport à des «formations discursives» distinctes, non seulement dans le temps, mais dans l'épistémè qui les fonde. La première Déclaration fut proclamée par «les représentants du peuple français, constitués en Assemblée nationale », au tout début de la Révolution française, la seconde fut «adoptée et proclamée par l’Assemblée générale des Nations Unies », à l'issue de la deuxième guerre mondiale. On passe d'une proclamation concernant un seul pays, la France, qui rompt avec la royauté absolue de droit divin, à une déclaration « universelle»; aux «droits de l'homme et du citoyen » répondent les «droits de l'homme».

Au diptyque droits et devoirs, homme et citoyens, répond l'unicité de 1948, celle des droits et de l'homme. A l'Assemblée constituante française répond l'Organisation des Nations Unies, et à l'exaltation révolutionnaire la victoire sur le nazisme, mais aussi la guerre meurtrière et la découverte des camps de déportation et d'extermination. Autres temps, autres Déclarations : ce sont l'homme et ses droits (inaliénables) qui font l'objet de la Déclaration universelle: 
C'est au juriste français René Cassin que l'on doit la qualification d' 'universelle' attribuée à la Déclaration qui faillit n'être qu'internationale. Il voulait ainsi souligner que les droits affirmés ne concernaient pas des citoyens, ressortissants d’États, mais des individus appartenant à une même fratrie. [...] La logique de la déclaration n'est pas celle d'un législateur, fût-il international, et de ses mandants, fussent-ils les cinquante-six Etats membres de l'époque. Elle est celle des victimes, où qu'elles se trouvent. (M. Bettati et O. Duhamel, 1998).

À des fondements et objectifs différents correspondent des Déclarations distinctes dans le temps; elles n'en sont pas moins en relation discursive, la seconde "parlant d'après » la première, et pouvant ainsi être appréhendée comme "modalisée par discours autre »(J. Authier-Revuz 2001). De surcroît leur dénomination commune, Déclaration, garde en 1948 la même caractérisation qu'en 1789: classée comme «spécialisé » et administratif par le «Grand Robert», elle appartient aux discours officiels et politiques et est en relation sémantique avec Proclamation. En atteste l'exemple retenu par le même dictionnaire : Déclaration ministérielle, " par laquelle le président du Conseil désigné par le président de la République indique les lignes générales de son programme devant l'Assemblée Nationale ». Les autres exemples donnés réfèrent tous à des actes civiques et/ou étatiques, comme «la Déclaration de guerre». On peut aussi y voir une trace de son emploi au $\mathrm{XVII}^{\circ}$ siècle comme «manifestation publique» (J. Dubois, R. Lagane 1960). La valeur officielle et solennelle vaut tant en 1789 qu'en 1948.

Après avoir analysé les droits garantis en 1789, et le diptyque, philosophique et juridique, des droits et devoirs, mais aussi de l'homme et du citoyen, notamment dans leur relation à la philosophie des Lumières et au «constitutionnalisme » anglais et américain, j'examinerai les droits de l'homme» dans la Déclaration de 1948, pour aborder enfin la « mémoire discursive » à l'œuvre.

\subsection{La Déclaration des droits de l'homme et du citoyen}

M. Vovelle (1970) nous rappelle :

à cette Révolution qui la première a tenté de modeler le réel sur un idéal formulé en termes d'universalité, il convient de laisser la parole pour proclamer les nouvelles vérités. Les textes majeurs prennent corps dès les premiers mois de la Révolution. C'est le 26 août 1789 que l'Assemblée constituante adopte le texte de la Déclaration des droits de l'homme et du citoyen. La Constitution de 1791 donne à ce texte de portée universelle prolongements et schémas d'application.

D'emblée, la Déclaration lie droits et devoirs, homme et citoyen: le Préambule rappelle «à tous les membres du corps social 'leurs droits et leurs devoirs'». Ce que précise l'article 4 de la Déclaration :

La liberté consiste à pouvoir faire tout ce qui ne nuit pas à autrui : ainsi, l'exercice des droits naturels de chaque homme n'a de bornes que celles qui assurent aux autres 
membres de la société la jouissance de ces mêmes droits. Ces bornes ne peuvent être déterminées que par la loi.

Omniprésente, la loi s'applique à tous, garantissant ainsi égalité et liberté, et s'opposant au bon vouloir royal. La Déclaration signe le remplacement du royaume, «État gouverné par un roi » (Dict. Robert, 1966) par le loyaume, néologisme avancé par quelques Révolutionnaires et cité par D. Corbin, opposant à la volonté d'un seul le règne de la loi promulguée par les (représentants des) Citoyens; l'antonymie qui oppose les deux termes est implicite dans la précision apportée par le «Dictionnaire Historique de la langue française » (1993), à la fin de l'article Royaume: «Royaume est employé abstraitement en parlant du domaine dans lequel une personne exerce sa prééminence (1643) [...].».

Le couple formé par les droits et devoirs est ainsi étayé. Reste à voir ce qui le définit, comme celui constitué par l'homme et le citoyen, et, plus largement, le fonctionnement et les valeurs tant de la synapsie les droits de l'homme que de la séquence peu ou prou figée dans le discours politique français, l'homme et le citoyen.

Rappelons d'autre part que l'emploi du présent confère une valeur générale, et non circonstancielle, aux principes qui sont énumérés, et tranche avec son «introduction» qui situe dans le temps les travaux des «représentants du peuple français » :

Les représentants du peuple français, constitués en Assemblée nationale, considérant que [...], ont résolu d'exposer dans une déclaration solennelle, les droits inaliénables et sacrés de l'homme. [Les soulignements sont de mon fait].

\subsection{Les droits de l'bomme}

On doit, semble-t-il, l'expression à Voltaire, dont, d'ailleurs, l'un des pamphlets a justement pour titre Les droits de l'homme (1768) :

Être libres, c'est raisonner juste, c'est connaitre les «droits de l'homme»; la loi d'Angleterre sur cette question ne mérite-t-elle pas de servir d'exemple à tous les législateurs qui voudront faire jouir l'homme des «droits de l'homme»? (Histoire de la langue française, VI, Première Partie 1, 1966).

Turgot, lui, proclame que «les 'droits des hommes' réunis en société ne sont pas fondés sur leur histoire, mais sur leur nature » (1775); d'autres parlent des « droits de l'humanité ». (ibid.). Quant aux Économistes du XVIII siècle, ils revendiquent aussi ces droits ; ainsi : «cette liberté sacrée, que l'on peut regarder comme le résumé de tous les droits de l'homme» (ibid.) ; dans les Notes à Quesnay, Dupont de Nemours rappelle que «la première notion des 'droits imprescriptibles de l'homme' était oubliée » (Notes à Quesnay).

Homme, depuis la fin du Xe siècle «s'emploie au sens d'être humain' (mâle ou femelle) [...], depuis le XII ${ }^{\mathrm{e}}$ s. [il] se dit d'un être humain considéré dans les qualités et les défauts propres à la nature humaine » (Le Robert, 1993). Homme désigne bien ainsi l'homme et la femme dans le vocabulaire révolutionnaire. Sa valeur de générique a 
sans doute facilité la fixation et la diffusion de la synapsie, couramment revendiquée tout au long de la seconde moitié du XXe siècle, et à l'origine du droit d'ingérence ; mais aussi celles des droits des travailleurs, droits des femmes, droits des enfants, droits des handicapés, etc., qui, par le double emploi du pluriel et d'hyponymes d'homme, synonyme de membre de l'bumanité, recourent à l'énumération et à la spécificité, là où les Révolutionnaires posent l'unicité abstraite. Abstraction et spécificités organisent partiellement la Déclaration de l'Indépendance des États-Unis (4 juillet 1776), « discours autre » modalisant la Déclaration française :

Nous tenons pour évidentes par elles-mêmes les vérités suivantes: tous les hommes sont créés égaux; ils sont doués par le Créateur de certains droits inaliénables; parmi ces droits se trouvent la vie, la liberté et la recherche du bonheur. Les gouvernements sont établis par les hommes pour garantir ces droits, et leur juste pouvoir émane des gouvernés.

L'égalité et la liberté, "droits naturels, inaliénables et sacrés » de l'homme, sont le fondement des autres droits reconnus par les textes institutionnels. En France, la Déclaration de 1793 y ajoutera des droits économiques et sociaux.

\section{Les droits}

Dans cette «Déclaration », discours officiel émanant d'une instance représentative de la Nation, le vocable droit(s), terme juridique, a aussi valeur politique. Au XVIII siècle, le substantif singulier droit a déjà pris l'un des sens d'aujourd'hui :

attesté au $\mathrm{VI}^{\circ}$ siècle au sens général de « justice, application de la loi », puis au VIII siècle, au sens de «règles, ensemble des lois ». [...] «Dès 1080, il est employé couramment, au singulier comme au pluriel, [droits] à propos de ce qui est permis ou exigible selon les principes d'une morale ou d'une législation [...] Au sens « comptable» (un, des droits) le mot entre dans de nombreux syntagmes, dont les droits de l'homme (1774) et la locution courante avoir le droit de...qui s'ajoute à avoir droit à... avec une autre nuance. (Le Robert (bistorique), 1993).

Il fonctionne avec une double référence, à la morale du droit naturel, qui fonde l'égalité des hommes (article premier), et à la (nouvelle) législation, que constitue la promulgation des droits naturels, inaliénables et sacrés de l'homme. Il est en relation avec le 'bien public' :

afin que cette déclaration, constamment présente à tous les membres du corps social leur rappelle sans cesse leurs droits et leurs devoirs; afin que les actes du Pouvoir législatif et ceux du Pouvoir exécutif, pouvant être à chaque instant comparés avec le but de toute institution politique [...] tournent toujours au maintien de la Constitution et au bonheur de tous. [...]. En conséquence, l'Assemblée nationale reconnaît et déclare, en présence et sous les auspices de l'Être suprême, les droits suivants de l'homme et du citoyen. [Les caractères gras sont de mon fait]. 
Cette complexité référentielle est notamment explicitée par l'emploi du syntagme de l'bomme et du citoyen. Les deux premiers articles définissent ses droits:

Article Premier. - Les hommes naissent et demeurent libres et égaux en droits. [...] Art. 2. - [...]. Ces droits sont la liberté, la propriété, la sûreté et la résistance à l'oppression.

La Constitution de 1793 y ajoutera des droits économiques et sociaux.

La devise républicaine et révolutionnaire, Liberté, égalité, fraternité, puise dans l'article premier et prend valeur de résumé des relations entre les citoyens, garanties par le respect de la loi.

Art. 9. - Tout homme étant présumé innocent jusqu'à ce qu'il ait été déclaré coupable, $[\ldots]$

Art. 10. - Nul ne doit être inquiété pour ses opinions, même religieuses, pourvu que leur manifestation ne trouble pas l'ordre public [...]

Art. 11. - La libre communication des pensées et des opinions est un des droits les plus précieux de l'homme ; tout citoyen peut donc parler, écrire, imprimer librement, $[\cdots]$

Aux droits naturels de l'homme, droits naturels et inaliénables chers aux philosophes des Lumières se combinent les droits politiques et institutionnels (dont le droit d'expression et d'opinion) qui en découlent, dans un État soucieux de respecter et de protéger les premiers : à la Constitution et aux lois de les garantir.

\subsection{L'bomme ET le citoyen}

Les deux syntagmes nominaux sont coordonnés dans l'intitulé de la Déclaration. La coordination des droits de l'homme et du citoyen est, bien évidemment, à mettre en relation avec les apports de la "philosophie des Lumières ». "L'effet de globalisation» opéré par la conjonction de coordination ET a déjà été signalé par J. Guilhaumou et D. Maldidier (1990), notamment dans l'expression, révolutionnaire, du pain ET la liberté, qui permet de "quitter le terrain du sensible pour accéder à la construction d'un savoir nouveau", ce qui est aussi le cas pour la lexie de la Déclaration: l'homme, entité philosophique et individu représentatif de l'espèce, est inséparable du citoyen, membre (actif) du corps social. Mais la relation qui unit les deux termes n'est pas symétrique : si être citoyen implique être homme, l'inverse n'est pas vrai. Deux conditions distinctes sont donc réunies, être homme, être (conjointement) citoyen, pour que soit valide la Déclaration de 1789.

Selon le Robert historique, c'est au XVII e siècle que citoyen « s'est spécialisé dans le vocabulaire politique au sens de 'membre d'une communauté politique organisée', d'abord par référence au modèle civique ancien et aux concepts romains de civis, civitas. ». Le dictionnaire cite le citoyen $d u$ monde de Voiture. L'Histoire de la langue française en précise l'emploi au XVIII' siècle :

le mot s'est acclimaté dans le vocabulaire des institutions françaises, répandu par les philosophes, notamment Rousseau (le citoyen de Genève), puis par la Révolution de 
1789, à la suite de laquelle le mot fut adopté comme titre et appellatif pour remplacer monsieur, madame (1790) [...] le mot citoyen entre dans les voies nouvelles. Le vieux sens de citoyen se trouve encore, mais rarement. [...] Rousseau a fixé les noms des Membres du Corps politique: "Ils prennent collectivement le nom de Peuple et s'appellent en particulier Citoyens, comme participant à l'autorité souveraine, et Sujets, comme soumis aux lois de l'Etat. [...]». (Contrat Social). [...]. Dans l'usage, en France, au nom de citoyen s'attachait de plus en plus l'idée de certains droits, mais surtout de certains devoirs envers la collectivité. [...]. L'Encyclopédie (1763), dans un long article, étudie à quelles conditions on est citoyen, c'est-à-dire «membre d'une société libre de plusieurs familles, qui partage les droits de cette société et qui jouit de ses franchises $»$.

Condorcet, dans L'esclavage des nègres, plaidait ainsi en faveur des protestants : "Qui est-ce qui ose se plaindre en France de la cruauté avec laquelle les protestants français sont privés des ‘droits de l'homme et du citoyen' ? (De l'esclavage des nègres) ; et Mably, en 1789, écrit Droits et devoirs du citoyen.

Si les droits naturels caractérisent l'bomme, le citoyen est donc celui qui participe aux affaires publiques et est défini par la loi comme membre d'un état de droit. Ce que J. Morange (1988) commente ainsi :

Ces deux qualités sont liées. [...]. Ici deux traditions se rejoignent : l'héritage antique pour lequel la liberté est essentiellement celle du citoyen ; [...] leurs traits modernes dans l'histoire pragmatique de l'Angleterre.

S'interrogeant sur la modernité politique, D. Schnapper (2000) rappelle qu'elle est

l'élaboration d'une société dans laquelle la citoyenneté constitue le fondement de la légitimité politique [...] Par la proclamation de cette nouvelle légitimité se trouvait posé un principe de transcendance des particularismes. Constitués en " nation », pour reprendre les termes de la Déclaration, les citoyens cessaient d'être des individus concrets, caractérisés par leurs origines historiques, leurs croyances et leurs pratiques religieuses, leurs appartenances sociales. Ils devenaient des citoyens égaux. Malgré les préjugés de l'époque, la citoyenneté fut étendue aux juifs en septembre 1791. Le nouveau principe de légitimité affirmait l'égalité civile, juridique et politique d'individus divers et inégaux. [le soulignement est de notre fait].

La citoyenneté serait ainsi la condition nécessaire à l'égalité des hommes ; c'est elle qui permet de passer du principe philosophique à son exercice. A cet égard, il n'est sans doute pas inutile de rappeler que l'auteur du décret reconnaissant aux juifs habitant en France le statut de citoyens, l'abbé Grégoire, fut aussi à l'origine de la première abolition de l'esclavage dans les colonies françaises, au nom des droits (de l'homme). Cependant, si les femmes appartiennent à la catégorie homme, la citoyenneté comprend des degrés : la Révolution leur reconnaît la citoyenneté publique (droit de réunion et de manifestation), mais pas la citoyenneté politique (droit de vote) et, en 
1795, leur interdit toute réunion publique et tout rassemblement dans la rue dépassant cinq participantes (M. Rebérioux et N. Savy, 1998). Les différents textes législatifs, dont les différentes Constitutions de la Révolution, définiront donc les droits distincts des citoyens et des citoyennes. Le citoyen visé par la Déclaration est sans doute bien cet «idéal modelant le réel en termes d'universalité » évoqué par M. Vovelle, mais non encore une réalité généralisée.

\subsection{Les droits du citoyen}

[lls] ne sont pas moins souvent proclamés : «les citoyens ont des droits, et des droits sacrés pour le corps même de la société » (Turgot, 1759, E Euvres). [...]. Nul doute que celle [la déclaration] de la Convention Américaine (1er juillet 1776) n'ait aidé à la diffusion de la formule. (Histoire de la langue française).

Parmi les Amendements à la Constitution des États-Unis, ces quelques extraits sont la preuve que l'expression est disponible pour les représentants du peuple français

1er amendement (1791). - Le Congrès ne pourra faire aucune loi concernant l'établissement d'une religion ou interdisant son libre exercice, restreignant la liberté de parole ou de la presse, ou touchant au droit des citoyens de s'assembler paisiblement $[\ldots]$.

4 ìme amendement (1791). - Le droit des citoyens d'être protégés dans leurs personnes, leurs maisons, leurs papiers et leurs effets et d'être mis à l'abri de toutes perquisitions et saisies déraisonnables $[\ldots]$.

Les articles 5, 6, 7, 8, 9, 10 et 11 de la Déclaration explicitent les domaines de la loi, les articles suivants impliquant sa prise en compte.

Article premier. - [...]. Les distinctions sociales ne peuvent être fondées que sur l'utilité commune.

Art. 2. - Le but de toute association politique est la conservation des droits naturels et imprescriptibles de l'homme. [...]

Art. 3. - Le principe de toute souveraineté réside essentiellement dans la Nation. [...]

Art. 5. - La loi n'a le droit de défendre que les actions nuisibles à la société. [...]

Art. 6. - La loi est l'expression de la volonté générale. Tous les citoyens ont droit de concourir personnellement, ou par leurs représentants, à sa formation. Elle doit être la même pour tous $[\ldots]$

L'ensemble de l'article premier énonce le postulat fondateur de la Déclaration; les suivants posent les principes et les conditions de l'exercice des droits; et c'est la complémentarité dialectique des droits et des devoirs qui en garantit l'exercice pour tous. La paire ainsi fondée résout le paradoxe soulevé par Voltaire dans l'article «Égalité » de son Dictionnaire philosophique: «L'égalité est donc à la fois la chose la plus naturelle et en même temps la plus chimérique. ». C'est la loi (commune) qui dit les devoirs civiques. 


\subsection{Les devoirs}

Le terme en tant que tel n'apparaît que rarement; mais les modaux devoir et pouvoir (à la forme négative) présentent un certain nombre d'occurrences, définissant et limitant les droits et précisant le rôle de la loi, « expression de la volonté générale »:

Art. 6. - [...] Elle [la loi] doit être la même pour tous, soit qu'elle protège, soit qu'elle punisse.

Art. 7. - Ceux qui sollicitent, expédient, exécutent ou font exécuter des ordres arbitraires doivent être punis ; mais tout citoyen, appelé ou saisi en vertu de la loi, doit obéir à l'instant; il se rend coupable par la résistance.

Art.8. - La loi ne doit établir que des peines strictement et évidemment nécessaires $[\ldots]$

Art. 9. - Tout homme étant présumé innocent [...] toute rigueur qui ne serait pas nécessaire pour s'assurer de sa personne doit être sévèrement réprimée par la loi. Art. 13. - [...] une contribution commune est indispensable: elle doit être également répartie entre tous les citoyens, en raison de leur faculté.

Art. 14. - Tous les citoyens ont le droit de constater [...] la nécessité de la contribution publique $[\ldots]$ aux citoyens.

On le constate, la loi et l'intérêt commun dictent des obligations (des devoirs)

Les occurrences négatives de pouvoir explicitent aussi les limites des droits et l'exercice de la citoyenneté :

Art.3. - [...] Nul corps, nul individu ne peut exercer d'autorité qui n'en [La Nation] émane expressément.

Art. 4. - Ces bornes [de l'exercice des droits naturels] ne peuvent être déterminées que par la loi.

Art. 5. - [...] Tout ce qui n'est pas défendu par la loi ne peut être empêché, et nul ne peut être contraint à faire ce qu'elle n'ordonne pas.

Art. 7.- Nul homme ne peut être accusé, arrêté ni détenu que dans les cas déterminés par la loi $[. .$.

Art. 8. $-[\ldots]$ nul ne peut être puni qu'en vertu d'une loi établie et promulguée $[\ldots]$

Art. 17. - La propriété étant un droit inviolable et sacré, nul ne peut en être privé, si ce n'est lorsque la nécessité publique, légalement constatée, l'exige [...]

La promulgation des droits de l'homme et du citoyen, mais aussi de ses devoirs découlant du bien fondé de la loi, et de la connaissance du domaine d'exercice. A cet égard, les 17 articles de la Déclaration de 1789 développent le Préambule : la dénonciation de «l'ignorance, l'oubli ou le mépris des droits de l'homme [qui] sont les seules causes des malheurs publics et de la corruption des gouvernements », le rappel des «droits» et « devoirs ». Depuis Montesquieu, les écrivains français du XVIII siècle ont célébré les vertus des lois et constitutions, et dénoncé l'arbitraire royal. La recherche du 
maintien de la Constitution » et «du bonheur de tous » se fonde ici sur la contrepartie des droits de l'homme et du citoyen, c'est-à-dire ses devoirs envers le corps social et politique auquel il appartient, la Nation. C'est en effet au XVIIIe siècle qu'émerge

\begin{abstract}
la notion de Nation : avec la Révolution, Nation devient une entité politique identifiée au Tiers état (Sieyès), au peuple révolutionnaire, et prend sa définition de «personne juridique constituée par l'ensemble des individus composant l'État» (arrêté du 23 Juillet 1789) (Dictionnaire historique de la langue française, 1993)
\end{abstract}

\title{
2. De 1789 a 1948 : des droits de l'homme et $d u$ citoyen aux droits de l'homme
}

Les intitulés des deux Déclarations sont construits sur le même schème La déclaration des droits de l'homme, mais diffèrent par la portée : de l'homme et du citoyen / de l'homme. Celle de 1789 valait pour la France, même si elle avait vocation implicite à une application à tous les peuples, celle de 1948 est universelle.

C'est au juriste français René Cassin que l'on doit la qualification d'« universelle» attribuée à la Déclaration qui faillit n'être qu'internationale. Il voulait ainsi souligner que les droits affirmés ne concernaient pas des citoyens, ressortissants d'États, mais des individus appartenant à une même fratrie. [...] La logique de la Déclaration n'est pas celle d'un législateur, fût-il international, et de ses mandants, fussent-ils les cinquante-six États membres de l'époque. Elle est celle des victimes, où qu'elles se trouvent. (M. Bettati et O. Duhamel, 1998).

Ces différences n'en font que mieux entendre des échos de 1948 répondant à 1789 , en en transformant l'héritage.

\subsection{Le Préambule}

Il intègre dans son propre discours comme fragments de discours autre (J. Authier, 1992) des extraits des « considérants » de 1789, sans expliciter ce discours autre modalisateur :

1789 Les représentants du peuple français, constitués en Assemblée nationale, considérant que l'ignorance, l'oubli ou le mépris des droits de l'homme sont les seules causes des malheurs publics et de la corruption des gouvernements, ont résolu d'exposer dans une déclaration solennelle les droits naturels, inaliénables et sacrés de l'homme, afin que cette déclaration, constamment présente à tous les membres du corps social, leur rappelle sans cesse leurs droits et leurs devoirs ; [...]

$\underline{1948}$ Considérant que la reconnaissance de la dignité inhérente à tous les membres de la famille humaine et de leurs droits égaux et inaliénables constitue le fondement de la liberté, de la justice et de la paix dans le monde,

Considérant que la méconnaissance et le mépris des droits de l'homme ont conduit à des actes de barbarie qui révoltent la conscience de l'humanité et que l'avènement d'un monde où les êtres humains seront libres de parler et de croire, libérés 
de la terreur et de la misère, a été proclamé comme la plus haute aspiration de l'homme, $[\ldots]$.

D'emblée se lisent deux mémoires, celle qui s'articule dans le temps court, celui de l'immédiat après la seconde guerre mondiale et qui se déploie dans l'énonciation de l'ONU, et celle qui s'articule dans le temps long et tisse les relations entre les deux discours. Mises en gras par nos soins, les «paroles » de 1948 « reprennent » celles du discours de 1789. A ces reprises s'articulent des variations et des différences.

Un même écho se lit dans le deuxième énoncé de l'article 1er de 1948, qui sonne comme un salut à la devise républicaine française :

Tous les êtres humains naissent libres et égaux [...]. Ils [...] doivent agir les uns envers les autres dans un esprit de fraternité.

\subsection{Les variations et les ajouts}

L'article 1er des deux Déclarations présente variations et ajouts :

$\underline{1789}$ Les hommes naissent et demeurent libres et égaux en droits. [...]

1948 Tous les êtres humains naissent libres et égaux en dignité et en droits. [...]

La relation de synonymie fonctionne dans la variante les hommes et les êtres humains. Le même article distingue deux emplois de droit: avoir le droit de, c'est-à-dire « avoir la possibilité, le pouvoir, ou l'autorisation », selon le Petit Robert, ce qui implique l'intervention d'une autorité, ici celle de la Nature (les droits naturels) et de la Loi ; et avoir droit à qui est plus centré sur le locuteur qui revendique. Le quantificateur tous réfère au Préambule et à la barbarie nazie.

Ces précisions inaugurent la série des droits que reconnaittra l'ONU : droits des femmes, des enfants, des handicapés, des minorités, ..., privilégiant l'énumération et non le générique, contrairement à la déclaration de 1789. Homme perd dès lors sa valeur d'hyperonyme pour ne plus désigner que l'hyponyme « être humain mâle ». La précision affaiblit le principe général.

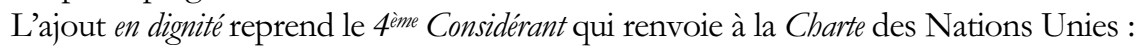

Considérant que dans la Charte les peuples des Nations unies ont proclamé à nouveau leur foi dans les droits fondamentaux de l'homme, dans la dignité et la valeur de la personne humaine $[\ldots]$,

et réfère implicitement au nazisme récusant le nom d'homme aux membres des « sousraces » (juive, tsigane, ...) et des « dégénérés » (homosexuels, handicapés, marxistes, ...), et prônant la «solution finale »; l'ONU réaffirme l'égalité «naturelle » des hommes et condamne tout racisme. L'histoire proche, celle du temps court d'une énonciation, fonde l'usage du vocable dignité. Ce qu'explicite l'article 2. :

Chacun peut se prévaloir de tous les droits et de toutes les libertés proclamés dans la présente Déclaration, sans distinction aucune, notamment de race, de couleur, de sexe, de langue, de religion, d'opinion politique ou de toute autre opinion, d'origine nationale ou sociale, de fortune, de naissance ou de toute autre situation. 
L'égalité de tous les êtres humains et de leurs droits est ainsi proclamée à partir des diversités humaines que les Révolutionnaires subsumaient par l'imposition d'un homme abstrait.

\subsection{L'énumération}

L'effacement du demeurent (1789, art.1.) est lié à l'énumération des bénéficiaires des droits et à celle de ces droits qu'on lit notamment dans la suite des articles; la Déclaration en comporte 30, souvent sub-divisés ; ainsi :

le droit à la vie, à la liberté et à la sûreté de sa personne, et droit sans distinction à une égale protection de la loi, droit à un recours effectif devant les juridictions nationales compétentes, droit de circuler librement et de choisir sa résidence à l'intérieur d'un Etat, droit de quitter tout pays, le droit de chercher asile, droit à une nationalité, droits égaux au regard du mariage, etc.

À ce long catalogue de droits privés et publics s'ajoutent quelques interdits et quelques limitations, qu'explicite notamment l'article 29.3. :

Ces droits et libertés ne pourront, en aucun cas, s'exercer contrairement aux buts et aux principes des Nations unies.

Là où 1789 recourt au générique l'homme, la loi, etc., 1948 énumère, ce qui « assigne à la séquence concernée une place dans la série » (Grammaire méthodique du français). La Déclaration française privilégiait la «compréhension » en indiquant les propriétés «que les éléments de l'ensemble présentent nécessairement» (Dictionnaire de linguistique), regroupant dans l'intitulé théorique «droits de l'homme» l'ensemble des droits reconnus aux membres de la société, et des devoirs qu'ils impliquent; celle de 1948 retient l'énumération des attributs (bomme $(s)$, femme $(s), \operatorname{enfant}(s), \ldots)$ et des différents droits découlant de la liberté et de l'égalité, ajoutant notamment parmi les "attributs» de l'homme la dignité, la paix, la personnalité juridique et le progrès social, recourant ainsi à l'« extension ».

Pour E. Decaux (1998), membre suppléant de la sous-commission des droits de l'homme des Nations unies depuis 1994 :

énumérer, c'est classer. Toute énumération implique une part d'amalgame. [... ]. Face à l'approche anglo-saxonne énumérant pêle-mêle les catégories défavorisées, le débat français sur la «parité » traduit bien l'actualité de cette problématique. Enfin, énumérer, c'est oublier. Malgré toutes ses précautions de style, la Déclaration comporte des omissions.

\section{4. «La non-discrimination»}

Le principe en est explicité par l'article 2. :

Chacun peut se prévaloir de tous les droits et de toutes les libertés proclamés dans la présente Déclaration, sans distinction aucune, notamment de race, de couleur, de sexe, de langue, de religion, d'opinion politique ou de toute autre opinion, d'origine nationale ou sociale, de fortune, de naissance ou de toute autre situation. 
De plus, il ne sera fait aucune distinction fondée sur le statut politique, juridique ou international du pays ou du territoire dont une personne est ressortissante [...].

Construit lui aussi par l'énumération, il présente, aux yeux d'E. Decaux,

le danger le plus grave [qui] est peut-être de nommer ce que l'on veut nier. La lutte contre la discrimination implique-t-elle de consacrer un vocabulaire connoté, qui passe par la référence à une race? (La déclaration universelle des droits de l'homme, ouvrage coll., p.34-36)

Le même auteur voit, dans cet article 2., qui ajoute à l'égalité

héritée de la tradition française, le principe de non-discrimination fortement marqué par une pratique anglo-saxonne qui préfère l'empirisme des garanties concrètes au lyrisme des idéaux révolutionnaires, [et oppose] l'égalité gravée pour l'éternité dans le marbre d'un universalisme abstrait, [à] la non-discrimination [qui] se décline dans le catégoriel.

Les principes langagiers et linguistiques mis en œuvre manifestent ainsi des choix différents tant dans l'énoncé des principes que de leurs applications; la prise en compte de la période historique et politique, ainsi que le rôle joué par les «Anglosaxons » pendant et après la guerre, participent aux «conditions de production du discours », le façonnent politiquement, juridiquement et textuellement.

La référence implicite au discours autre des précédentes Déclarations modalise le discours et le situe dans l'histoire tant des droits naturels de l'homme que des droits politiques et juridiques. C'est l'ONU qui peut seule garantir ces « droits universels », par des votes de condamnation de certains États et par les nombreuses « interventions humanitaires» qui jalonnent son existence. Les membres souverains de l'ONU s'engagent en effet :

1. A assurer la paix du monde par des moyens pacifiques (recommandations, enquêtes, médiations, arbitrages - art. 35), par des sanctions politiques ou économiques (art. 41), ou l'engagement des forces armées (art. 42) mises à leur disposition par les membres ;

2. A assurer le droit de défense des États (art. 51), même au moyen de pactes régionaux de sécurité ;

3. A remplir loyalement leurs obligations envers les Nations unies (art. 2), particulièrement à renoncer à la menace ou à l'emploi de la force. (in Atlas historique, 1997).

\subsection{Modalisation et situation dans le temps}

Le discours recourt volontiers aux modaux pouvoir (art.2, 9, 14.2., 16.2., 17.2., 20.2., 29.3., 30.) et devoir (art.1., 21.3., 26.2.) ; pouvoir implique la prise en compte des données réelles de réalisation pour les récuser : «Nul ne peut ... »; le second participe du discours de volonté surplombant et transcendant les données empiriques. Ce à quoi participe le futur, avec valeur d'injonction tempérée portant sur l'avenir ; on y lit une 
«nuance de règle morale », l'ordre strict n'ayant ici aucune chance perlocutoire d'être suivi d'effet par tous les États.

L'emploi du passé s'oppose soit au présent, soit au futur ; il peut stigmatiser un passé encore proche et rejeter des visées du discours des faits à exclure dans l'avenir. Le deuxième alinéa du préambule situe le discours tenu par rapport à ce passé proche, et le stigmatise au présent; il peut aussi être la toile de fond qui fonde positivement le discours et lui donne sens :

Considérant que dans la Charte les peuples des nations unies ont proclamé à nouveau leur foi dans les droits fondamentaux de l'homme [...].

S'opposant ainsi au discours généralisant de 1789, la Déclaration de 1948 proclame des droits d'ordre philosophique, valant urbi et orbi, dans une période historique et idéologique explicitement située, en rupture avec le totalitarisme nazi.

\section{6. "L'bomme » sans "le citoyen"}

La plus grande différence réside dans cette absence. Le discours institutionnel et principiel de 1789 pose les fondements d'un nouveau monde. Ce que reprennent les préambules des Constitutions françaises de la $\mathrm{IV}^{\circ}$ et de la $\mathrm{V}^{\circ}$ Républiques :

[...] Il [le peuple français] réaffirme solennellement les droits et les libertés de l'homme consacrés par la Déclaration des droits de 1789 [...] (27 octobre 1946),

Le peuple français proclame solennellement son attachement aux Droits de l'homme et aux principes de la souveraineté nationale tels qu'ils ont été définis par la Déclaration de 1789, confirmée et complétée par le préambule de la Constitution de $1946[\ldots]$ (4 octobre 1958).

Seuls «les droits de l'homme» sont invoqués dans les préambules, puisque « les droits du citoyen » relèvent des Constitutions nationales.

La Déclaration universelle de 1948 ne peut le faire, puisque les hommes visés par le discours ne sont définis que par leurs droits naturels, et non aussi par leurs rôles civiques. Elle proclame sa portée universelle en posant les mêmes droits quel que soit le statut politique, juridique ou international du pays ou du territoire dont une personne est ressortissante, que ce pays soit indépendant, sous tutelle ou soumis à une limitation quelconque de souveraineté (art. 2.).

D'autres articles (préambule, 21, 29, 30,) s'efforcent de rappeler (peuvent-ils l'imposer ?) le principe d'un État de droit et le refus de la dictature. Ses formulations humanitaristes aboutiront au principe d'intervention, manifesté par les « forces d'interposition » et, peu ou prou, par le droit d'ingérence, ressenti comme un devoir :

\section{1. - Toute personne a le droit de prendre part à la direction des affaires} publiques de son pays, $[\ldots]$

3. - La volonté du peuple est le fondement de l'autorité des pouvoirs publics ; cette volonté doit s'exprimer par des élections honnêtes qui 
doivent avoir lieu périodiquement, au suffrage universel égal et au vote secret $[\ldots]$

29. 1. - L'individu a des devoirs envers la communauté [...]

2. - Dans l'exercice de ses droits et dans la jouissance de ses libertés, chacun n'est soumis qu'aux limites établies par la loi exclusivement en vue d'assurer la reconnaissance et le respect des droits et libertés d'autrui et afin de satisfaire aux justes exigences de la morale, de l'ordre public et du bien-être général dans une société démocratique.

3. - Ces droits et libertés ne pourront, en aucun cas, s'exercer contrairement aux buts et aux principes des Nations Unies.

30. - Aucune disposition de la présente Déclaration ne peut être interprétée comme impliquant pour un État, un gouvernement ou un individu un droit quelconque de se livrer à une activité ou d'accomplir un acte visant à la destruction des droits et libertés qui y sont énoncés.

\section{D’une mémoire discursive a une autre}

Le débat, philosophique et logique, entre abstrait et concret, compréhension et extension, généricité et énumération se répercute dans une analyse des moyens linguistiques mis en œuvre dans deux discours à la fois proches et différents. Leur construction en articles, leurs intitulés et leur visée les relient par une (longue) mémoire discursive.

Puisant dans l'approche de M. Foucault (1966) qui relève ces «discours qui sont à l'origine d'un certain nombre de paroles qui les reprennent, les transforment en parlant d'eux [...]», J.-J. Courtine (1981) avance le terme mémoire discursive et parle d'effets de mémoire qui «s'articulent dans le temps long d'une mémoire [distincte de la mémoire retenue par les psycholinguistes], mais aussi dans le temps court de l'actualité d'une énonciation ». Ces effets de mémoire structurent notamment les deux intitulés, en leur conférant nom et caractère discursif. Mais là où les Révolutionnaires français ont avancé le néologisme vite disparu loyaume appelé à se substituer à royaume, les États membres de l'ONU en appellent à la nécessaire fraternité entre les hommes.

La mémoire discursive lointaine s'arrête dès lors; loin de l'optimisme humaniste des Lumières, l'ONU prend acte de l'histoire proche, pour postuler le droit à la liberté, quel que soit le régime politique des États où vivent les êtres humains, nés libres et égaux et pour préciser leur égale dignité. Là où les Révolutionnaires visaient «le maintien de la Constitution et [le] bonheur de tous", le Préambule de 1948 considère notamment "que l'avènement d'un monde où les êtres humains seront libres de parler et de croire, libérés de la terreur et de la misère, a été proclamé comme la plus haute aspiration de l'homme. ». Et c'est aussi une autre mémoire discursive, celle des écrits de Gobineau, Lapouge ou Le Bon (Taguieff 1998), celle des discours du führer Hitler, et celle des camps et des chambres à gaz, qui se lit dans la Déclaration universelle. Mais aussi l'espoir en un monde qui a su triompher de la barbarie et se regrouper en Nations unies. 


\section{BIBLIOGRAPHIE}

GANDINI, J.-J. (1998), Les droits de l'homme, anthologie, Librio.

La Déclaration universelle des droits de l'homme (1998), textes rassemblés par M. Bettati.

Duhamel, O., L. Greilsamer, Gallimard Folio actuel Nº 64, Paris.

Hilgemann, W., Kinder, H. (Albeck R. trad.) (1997), Atlas historique, Perrin, Paris.

SCHNAPPER, D., Qu'est-ce que la citoyenneté (2000), Gallimard Folio actuel N 75, Paris.

LE ROBERT (1966), Dictionnaire alphabétique et analogique de la langue française, Société du nouveau Littré Le Robert, Paris.

LE ROBERT (1993), Dictionnaire bistorique de la langue française, dir. A. Rey, Dictionnaires Le Robert, Paris.

Brunot, F. (1966), Histoire de la langue française, tome VI Le XVIII siècle Première Partie «Le mouvement des idées et les vocabulaires techniques », Fascicule premier, bibliographie établie par Deloffre F., Armand Colin, Paris.

Dubois, J. Et Lagane, R. (1960), Dictionnaire de la langue française classique, Librairie Belin, Paris.

DuBoIs, J. et al. (1973), Dictionnaire de linguistique, Dubois A. et al., Larousse, Paris.

Riegel, M., Pellat, J.-C., Rioul, R. (1994), Grammaire méthodique du français, PUF Linguistique nouvelle, Paris.

Authier-RevUZ, J. (2001), « La représentation du discours autre », « Modalisation par discours autre et bivocalité », in Une langue, le français, R. Tomassone dir., Hachette, Paris.

Courtine, J.-J. (1981), Analyse du discours politique, Langages N62, Larousse, Paris.

Courtine, J.-J. (1994), Mémoire, histoire, langage, Langages Nº114, Larousse, Paris.

Foucault, M. (1966), Les mots et les choses, Gallimard, Paris.

Guilhaumou, J., MALDidieR, D. (1990), « De nouveaux gestes de lecture ou le point de vue de l'analyse de discours sur le sens », La quadrature du sens, Normand C. dir., PUF, Nouvelle Encyclopédie Diderot, Paris.

Petiot, G. (1990), «Entre le consensus et les divergences : 'Liberté(s)' dans les discours politiques », La quadrature du sens, Normand C. dir., PUF, Nouvelle Encyclopédie Diderot, Paris.

Petiot, G. (2003), «Langue de bois et fait autonymique », Parler des mots Le fait autonymique en discours, PSN, Paris.

TAguiefF, P.-A. (1998), La couleur du sang Doctrines racistes à la française, Edit. Mille et une Nuits Les petits libres $\mathrm{N}^{\circ} 15$.

Tomassone, R. dir. (2001), Une langue, le français, Hachette Grands Repères Culturels, Paris. 
